\title{
Physical activity in sports clubs of children and adolescents in Germany: results from a nationwide representative survey
}

\author{
Darko Jekauc • Anne Kerstin Reimers • \\ Matthias Oliver Wagner • Alexander Woll
}

\begin{abstract}
Aim A substantial proportion of children's and adolescent's physical activity takes place in organised sports clubs in Germany. The purpose of this study was to describe (1) the proportion of children and adolescents who are members of sports clubs and the amount of moderate to vigorous physical activity (MVPA) performed in these clubs, and to identify socio-demographic predictors of (2) membership and (3) the amount of MVPA in sports clubs in Germany.

Subjects and methods The sample consisted of 4,529 boys $(50.5 \%)$ and girls (49.5\%) aged between 4 and 17 years $(M=11.3 ; \mathrm{SD}=4.1)$. MVPA was assessed by a questionnaire. Socioeconomic status, immigration background, and residential area were assessed using a parent questionnaire. Results $52.0 \%$ of the girls and $63.1 \%$ of the boys were members in sports clubs who exercised on average for $4 \mathrm{~h}$ per week with moderate to high intensity. The logistic regression analyses showed that gender, socioeconomic status, migration
\end{abstract}

D. Jekauc $(\bowtie) \cdot$ M. O. Wagner

Department of Sport Science, University of Konstanz, Universitätsstraße 10, 78457 Constance, Germany

e mail: darko.jekauc@uni konstanz.de

M. O. Wagner

e mail: matthias.wagner@uni konstanz.de

\section{A. K. Reimers}

Department of Sport Science, University of Konstanz,

Universitätsstraße 10, 78457 Constance, Germany

e mail: anne.reimers@uni konstanz.de

\section{A. K. Reimers}

Department of Sport Science, Karlsruhe Institut for Technology, Engler Bunte Ring 15, Geb. 40.40,

76131 Karlsruhe, Germany

\section{A. Woll}

Department of Sport Science, Karlsruhe Institut for Technology,

Engler Bunte Ring 15, Geb. 40.40, Raum 108,

76131 Karlsruhe, Germany

e mail: alexander.woll@kit.edu background and residential area were significant predictors of membership in sports clubs. The amount of MVPA was only significantly predicted by age, gender and residential area.

Conclusion The results of this study imply that sports clubs have a high potential for implementation of strategies to increase general levels of physical activity.

Keywords Physical activity · Sports club · Children · Adolescents · Germany

\section{Introduction}

The health benefits of physical activity are well documented (Bouchard et al. 2012). Especially in children and adolescents, regular physical activity is associated with improved psychological well-being and lower levels of cardiometabolic risk factors (Strong et al. 2005). According to physical activity guidelines (WHO 2010) physical activity levels of children and adolescents in Germany are believed to be insufficient. Only $17 \%$ of boys and $13 \%$ of girls aged between 4 and 17 years meet the physical activity guideline (Jekauc et al. 2012). Furthermore, low levels of physical activity seem to persist into adulthood (Telama et al. 2005).

In Germany, a substantial proportion of children's and adolescent's physical activity takes place in organised sports clubs (Brettschneider 2001). These sports clubs are organisations that have the primary mission of facilitating participation in an activity that involves both physical and mental components in the pursuit of recreation and/or competition (Heinemann 1999). Out of 90,000 existing sports clubs in Germany, 56,500 sports clubs offer a wide range of activities in different types of sport for preschoolers and 84,000 for schoolchildren and adolescents (Breuer and Wicker 2009). In contrast to Anglo-American regions, sports clubs are available in most parts of Germany, even in rural areas (Baur and Burrmann 2000). Membership in sports clubs is affordable 
for the majority of the German population, thus providing an opportunity for most Germans to be physically active. Therefore, sports clubs in Germany have considerable potential to raise the general level of physical activity, and are well suited for nationwide health promotion interventions, as shown in other countries (e.g. Kokko et al. 2009). In addition, sports clubs were identified as a means for introducing health related policies (Dobbinson et al. 2006). Brettschneider (2001) showed that adolescent's membership in sports clubs is associated with higher performance in speed, strength, endurance and coordination tests and with higher ratings in self-concept and psycho-social health when compared to adolescents who are not a member of a sports club. Therefore, being physically active in sports clubs can be seen as one of the most important sources of children's and adolescent's physical activity in terms of quantity and quality.

Despite the importance of sports clubs for children's and adolescent's development, representative data on physical activity in sports clubs and potential predictors of membership, and the amount of physical activity in sports clubs for children and adolescents in Germany are lacking. International studies showed that socio-demographic characteristics such as age, gender, social status or ethnicity are major predictors of physical activity in general (Van der Horst et al. 2007). However, because the role of physical activity in sports clubs is less important in Anglo-American countries, there is scarce evidence for prevalence and predictors of being physically active in sports clubs in international literature. In addition, previous German studies have several limitations: data for children younger than 11 years are scarce; previous studies concentrated on specific population groups (e.g. girls); and nonvalidated measures of physical activity were used. Therefore, the purposes of this study were to identify socio-demographic predictors of (1) the membership and (2) the amount of physical activity in sports clubs in Germany.

\section{Subjects and methods}

\section{Sampling and participants}

Participants of this study were selected from the Motorik Modul (MoMo-Study), a cross-sectional study that aims to estimate prevalence rates in physical activity and level of motor abilities in children and adolescents aged between 4 and 17 years in Germany (Woll et al. 2011). The sample of the MoMo-Study is a representative sub-sample of the German Health Interview and Examination Survey for Children and Adolescents (KiGGS) (Kurth et al. 2008). Oversampling procedures regarding age, gender, region, and country of citizenship were used to ensure the representativeness of the data. Participants of the MoMoStudy were recruited in three steps. First, a systematic sample of 167 primary sampling units was selected from an inventory of
German communities (Kurth et al. 2008). Second, an agestratified sample of randomly selected children and adolescents was drawn from the official registers of local residents for the KiGGS with a total of 17,641 participants aged between 0 und 17 years (Kamtsiuris et al. 2007). Third, 7,866 participants aged between 4 and 17 years in the KiGGS-sample were randomly assigned to the sample of the MoMo-Study. Of these 7,866 participants, 4,529 children and adolescents participated in the MoMo-Study (response rate $=57.6 \%$ ).

In accordance with this three-step recruitment process, weighting procedures were used to ensure the representativity of the results. In the first step, design weights were calculated which are the products of the inverse selection probabilities of each community and each participant within the community according to age and gender. In the second step, adjustment weighting was conducted to adjust the deviations of the design weighted net sample of the KiGGS from the population structure (31 December 2004) considering age, gender, region (East vs. West Germany) and nationality (German vs. not German; Kamtsiuris et al. 2007). In a third step, inverse selection probabilities were calculated to rule out deviations of the net sample of the MoMo-Study from the KiGGS net sample. A set of variables as age, gender, social status, migration background, residential area, physical activity, motor abilities, subjective and objective measures of health status were used to assess the deviations between the MoMo-Study and the KiGGS and to calculate the selection probabilities (Bös 2009). The results of these analyses showed that age, socioeconomic status, migration background and subjective health had a significant effect on participation rates (Bös 2009). The final weight was a product of these three-step weightings which was normed to the sample size of the MoMo-Study.

The KiGGS and the MoMo-Study were approved by the Charité/Universitätsmedizin Berlin ethics committee and the Federal Office for the Protection of Data and were conducted according to the Declaration of Helsinki (Kurth 2007). All participants and their guardians gave written informed consent prior to study participation. To ensure the representativeness of the study results, deviations of the sample from the population structure regarding age, gender, region and country of citizenship oversampling procedures were used (Kurth et al. 2008).

\section{Data collection}

Data were collected at central locations at the 167 sample points selected for the KiGGS and and the MoMo-Study. Data collection of the MoMo-Study followed approximately two weeks after the examination of the KiGGS-Study. Data collection took place between May 2003 and May 2006 (Woll et al. 2011). All potential participants of the MoMo-Study were asked by the personnel of the examiner team of the KiGGS for permission to be contacted by the team of the MoMo-Study. In the cases where permission was granted, 
the potential participants were firstly contacted by letter containing a pamphlet to provide basic information on the MoMoStudy and secondly by telephone to arrange an appointment at the examination location. In order to improve participation rate participants aged 10 years or younger received a small present (e.g. cuddly toy) and participants aged 11 years or older received an incentive of 10 Euro. Participants aged younger than 11 years were interviewed with assistance of their parents by qualified interviewers. Participants aged 11 years or older completed the physical activity questionnaire independently.

\section{Measurement}

\section{Physical activity in sports clubs}

Physical activity in sports clubs was assessed using the MoMo Physical Activity Questionnaire (MoMo-PAQ). The part of the questionnaire on physical activity in sports clubs consists of six questions on: (1) membership, (2) type of sports, (3) frequency of training sessions, (4) duration of training sessions, (5) seasonality (months in year), and (6) intensity. If participants practised more than one sport in clubs, the questions (2-6) were repeated for each sport. Based on the questionnaire, the time spent in moderate to vigorous physical activity (MVPA) in sports clubs was calculated as a composite index. The test-retest reliability of the measure was shown to be sufficient: for each item the Cohen's Kappa coefficient was significant with an average value of 0.81 for a one-week test-retest interval (Jekauc et al. 2013). In addition, the composite index for physical activity in sports clubs correlated significantly with accelerometer measured physical activity (Actigraph GT1M;r=0.35) and with physical activity measured using the Ppevious day physical activity recall (PDPAR; $r=0.55$; Jekauc et al. 2013).

\section{Socio demographic characteristics}

Socio-demographic characteristics were assessed using a parent questionnaire conducted as part of the KiGGS (Kurth et al. 2008). The questionnaire included items on both parents' educational and professional status, as well as on total income available to the family household (Winkler and Stolzenberg 1999). The categorisation of their socioeconomic status was conducted according to Winkler and Stolzenberg (1999). The migration background variable was constructed based on information on nationality, country of birth, year of immigration of both parents as well as historical criteria (e.g. labour migrants, asylum seekers), languages spoken at home and the proficiency in German of the children and parents (Kurth et al. 2008). The residential area variable reflected the level of urbanisation of the residential environment. Towns or settlements with less than 5,000 residents were classified as rural areas, towns with 5,000-19,999 residents as small towns, towns with 20,000-99,999 residents as medium-sized towns and cities with 100,000 or more residents as cities.

\section{Statistical analysis}

Data was analysed using the IBM Statistical Package for Social Science (SPSS) version 19 (IBM, New York, USA). All analyses were calculated separately for children (4-10 years) and adolescents (11-17 years), and for boys and girls, because our preliminary analyses showed that gender significantly interacted with residential area and socioeconomic status; whereas age significantly interacted with migration background and socioeconomic status. Descriptive statistics were calculated for all predictors and for the outcome variable. The two outcome variables were the binomial variable "membership in sports club" analysing physical activity participation in sports clubs and the amount of MVPA in sports clubs quantified by a composite index as a continuous variable. Participants' age, migration background, socioeconomic status and residential area were considered as potential predictors for both outcome variables. Bivariate and multivariate analysis strategies were used to rule out confounding effects. Chi-square goodness of fit tests and t-tests were performed to evaluate bivariate associations between the predictors and the outcome variables. Multiple logistic regressions were calculated to assess the incremental power of prediction of each predictor on physical activity participation in sports clubs. Goodness of fit was assessed using Nagelkerke Pseudo- $R^{2}$, which varies between 0 and 1 . A higher value of Nagelkerke Pseudo- $R^{2}$ indicates a better prediction of the model. Multiple linear regressions were used to analyse the influence of predictors on the amount of physical activity in sports clubs. Here, goodness of fit was assessed using the determination coefficient $R^{2}$ which varies between 0 and 1 and can be interpreted as the proportion of explained variance. Because all non-members have a constant amount of physical activity in sports clubs of zero, they were excluded from all multiple linear regression analyses and as a result, deviations from the normal distribution were avoided. For all four multiple linear regressions, Kolmogorov-Smirnov tests did not indicate significant deviations of the residuals from the normal distribution, which is a prerequisite of multiple regression analysis. To adjust $p$-values and confidence intervals for clustering effects within the primary sampling units, the SPSS-Module "Complex Samples" was used.

\section{Results}

\section{Sample characteristics}

Descriptive statistics of the sample are shown in Table 1. The proportion of boys $(50.5 \%)$ and girls $(49.5 \%)$ in the sample was almost equal. The mean age was 11.3 years 
Table 1 Frequency distributions for the potential predictors and the outcome variable

\begin{tabular}{lrrr}
\hline Variable & Total $(N=4,529)$ & Boys $(n=2,285)$ & Girls $(n=2,244)$ \\
\hline Age group & & & \\
45 years & $989(21.8 \%)$ & $484(21.2 \%)$ & $505(22.5 \%)$ \\
610 years & $1,712(37.8 \%)$ & $863(37.8 \%)$ & $849(37.8 \%)$ \\
11 13 years & $886(19.6 \%)$ & $470(20.6 \%)$ & $416(18.5 \%)$ \\
1417 years & $942(20.8 \%)$ & $468(20.5 \%)$ & $474(21.1 \%)$ \\
Socioeconomic status & & & \\
Low & $1,157(25.8 \%)$ & $578(25.6 \%)$ & $479(26.1 \%)$ \\
Medium & $2,195(49.0 \%)$ & $1,113(49.3 \%)$ & $1,082(48.7 \%)$ \\
High & $1,127(25.2 \%)$ & $568(25.1 \%)$ & $559(25.2 \%)$ \\
Migration background & & & $243(10.9 \%)$ \\
Yes & $496(11.0 \%)$ & $253(11.1 \%)$ & $1,988(89.1 \%)$ \\
No & $4,013(89.0 \%)$ & $2,025(88.9 \%)$ & $580(25.8 \%)$ \\
Residential area & & & $643(28.7 \%)$ \\
Rural area & $1,173(25.9 \%)$ & $593(26.0 \%)$ & $670(29.9 \%)$ \\
Small town & $1,334(29.5 \%)$ & $691(30.2 \%)$ & $351(15.6 \%)$ \\
Medium sized town & $1,315(29.0 \%)$ & $645(28.2 \%)$ & $1,146(52.0 \%)$ \\
City & $707(15.6 \%)$ & $356(15.6 \%)$ & $139.9(135.2)$ \\
Members in sports clubs & & & \\
Membership & $2,502(57.7 \%)$ & $1,450(63.1 \%)$ & \\
Member's MVPA & $161.1(140.1)$ & & \\
Mean (SD) & & & \\
\hline
\end{tabular}

$(\mathrm{SD}=4.1$ years). Almost half of the participants were classified as falling in the middle socioeconomic status category. A quarter of the sample was of high and a quarter of low socioeconomic status. Eleven percent of the participants had a migration background. While $25.9 \%$ of the participants lived in rural area, $58.5 \%$ were inhabitants of small and medium-sized towns.

Prevalence of membership and amount of physical activity in sports clubs

Overall, $57.7 \%$ of the participants were members in at least one sports club (Table 1). Members of sports clubs exercised on average for $161.1 \mathrm{~min}(\mathrm{SD}=140.1 \mathrm{~min})$ per week with moderate to vigorous intensity. A greater proportion of boys $(63.1 \%)$ were members in sports clubs than that of girls $\left(52.0 \% ; \chi^{2}=56.8 ; \mathrm{df}=1 ; p<0.001\right)$. Among members in sports clubs, boys were significantly more physically active (mean \pm 1 $\mathrm{SD}, 178.0 \pm 141.8 \mathrm{~min})$ than girls $(139.9 \pm 135.2 \mathrm{~min} ; t=7.1$; $\mathrm{df}=2,443 ; p<0.001)$.

Socio-demographic predictors of membership in sports clubs

Table 2 shows the results of the logistic regression analysis which evaluates the predictive power of the sociodemographic correlates of membership in sports clubs in children aged between 4 and 10 years. The proportion of explained variance was moderate with Nagelkerke Pseudo$R^{2}$ of 0.123 for boys and 0.158 for girls.
In children, socioeconomic status and migration background had a significant effect on membership in sports clubs in both boys and girls. The chance of being a member of a sports club was 3.6 times lower for boys and 3.0 times lower for girls with a low socioeconomic status than for boys and girls with a high socioeconomic status. Boys without a migration background had a $70 \%$ and girls 4.2 times higher chance of being a member of a sports club than boys and girls with a migration background. Age was only a significant predictor in boys. For boys, the chance of being a member of a sports club increased by $20 \%$ with every year of life. Residential area was a significant predictor but only in girls and not in boys. Girls living in small and medium-sized towns were approximately twice as likely to be members of a sports club than girls living in cities.

Table 3 shows the results of the logistic regression analyses for predicting membership in sports clubs for adolescents. The amount of explained variance was small with Nagelkerke's $R^{2}$ of 0.085 for boys and 0.105 for girls.

In adolescents, age and socioeconomic status were significant predictors of membership in sports clubs for boys and girls. The chance of being a sports club member decreased by $15 \%$ for boys and by $11 \%$ for girls with every year increase in age. The chance of being a sports club member for adolescents with a low socioeconomic status was 3.0 times lower than for adolescent boys and girls with a high socioeconomic status. Migration background and residential area were significant predictors only in adolescent girls but not in boys. Adolescent girls without a migration background had a $90 \%$ higher 
Table 2 Logistic regression for predicting membership in sports clubs in 410 year old children

\begin{tabular}{|c|c|c|c|c|c|c|c|c|c|c|c|c|}
\hline & \multicolumn{6}{|c|}{ Boys $^{\mathrm{a}}$} & \multicolumn{6}{|c|}{ Girls $^{\mathrm{b}}$} \\
\hline & \multirow[t]{2}{*}{$\chi^{2}$} & \multirow[t]{2}{*}{ df } & \multirow[t]{2}{*}{$p$} & \multirow[t]{2}{*}{ adj OR } & \multicolumn{2}{|c|}{$95 \%$ CI for OR } & \multirow[t]{2}{*}{$\chi^{2}$} & \multirow[t]{2}{*}{$\mathrm{df}$} & \multirow[t]{2}{*}{$p$} & \multirow[t]{2}{*}{$\operatorname{adj} \mathrm{OR}^{\mathrm{c}}$} & \multicolumn{2}{|c|}{$95 \% \mathrm{CI}^{\mathrm{d}}$ for $\mathrm{OR}$} \\
\hline & & & & & Lower & Upper & & & & & Lower & Upper \\
\hline Age (in years) & 28.5 & 1 & $<0.001$ & 1.20 & 1.12 & 1.29 & 0.5 & 1 & 0.494 & 1.02 & 0.96 & 1.10 \\
\hline Socioeconomic status & 48.1 & 2 & $<0.001$ & & & & 31.6 & 2 & $<0.001$ & & & \\
\hline Low & 43.6 & 1 & $<0.001$ & 0.28 & 0.19 & 0.41 & 31.4 & 1 & $<0.001$ & 0.33 & 0.22 & 0.49 \\
\hline Medium & 5.4 & 1 & 0.020 & 0.67 & 0.47 & 0.94 & 13.2 & 1 & $<0.001$ & 0.54 & 0.39 & 0.76 \\
\hline High (ref.) & & & & 1.00 & & & & & & 1.00 & & \\
\hline Migration background & 7.2 & 1 & 0.007 & 1.70 & 1.15 & 2.49 & 38.7 & 1 & $<0.001$ & 4.17 & 2.66 & 6.54 \\
\hline Yes (ref.) & & & & 1.00 & & & & & & 1.00 & & \\
\hline No & 7.2 & 1 & 0.007 & 1.70 & 1.15 & 2.49 & 38.7 & 1 & $<0.001$ & 4.17 & 2.66 & 6.54 \\
\hline Residential area & 2.5 & 3 & 0.478 & & & & 28.9 & 3 & $<0.001$ & & & \\
\hline Rural area & 0.0 & 1 & 0.845 & 1.04 & 0.69 & 1.58 & 0.6 & 1 & 0.437 & 0.85 & 0.56 & 1.28 \\
\hline Small town & 0.9 & 1 & 0.342 & 1.20 & 0.83 & 1.74 & 14.6 & 1 & $<0.001$ & 2.08 & 1.43 & 3.01 \\
\hline Medium sized town & 2.0 & 1 & 0.157 & 1.30 & 0.90 & 1.88 & 10.5 & 1 & 0.001 & 1.81 & 1.27 & 2.60 \\
\hline City (ref.) & & & & 1.00 & & & & & & 1.00 & & \\
\hline Intercept & 4.7 & 1 & 0.030 & 0.47 & & & 5.9 & 1 & 0.015 & 0.40 & & \\
\hline
\end{tabular}

a For boys: $N=1,347 ; 2 \log$ Likelihood $=1263.5$; Nagelkerke $R^{2}=0.132$

${ }^{\mathrm{b}}$ For girls: $N=1,322 ; 2 \log$ Likelihood $=1247.5$; Nagelkerke $R^{2}=0.158$

c adj ORadjusted odds ratio

${ }^{\mathrm{d}}$ CIconfidence interval

Table 3 Logistic regression for predicting membership in sports clubs in 1117 year old adolescents

\begin{tabular}{|c|c|c|c|c|c|c|c|c|c|c|c|c|}
\hline & \multicolumn{6}{|c|}{ Boys $^{\mathrm{a}}$} & \multicolumn{6}{|c|}{ Girls $^{\mathrm{b}}$} \\
\hline & \multirow[t]{2}{*}{$\chi^{2}$} & \multirow[t]{2}{*}{$\mathrm{df}$} & \multirow[t]{2}{*}{$p$} & \multirow[t]{2}{*}{$\operatorname{adj} \mathrm{OR}$} & \multicolumn{2}{|c|}{$95 \% \mathrm{CI}$ for OR } & \multirow[t]{2}{*}{$\chi^{2}$} & \multirow[t]{2}{*}{$\mathrm{df}$} & \multirow[t]{2}{*}{$p$} & \multirow[t]{2}{*}{$\operatorname{adj} \mathrm{OR}^{\mathrm{c}}$} & \multicolumn{2}{|c|}{$95 \% \mathrm{CI}^{\mathrm{d}}$ for $\mathrm{OR}$} \\
\hline & & & & & Lower & Upper & & & & & Lower & Upper \\
\hline Age (in years) & 27.5 & 1 & $<0.001$ & 0.85 & 0.80 & 0.90 & 13.8 & 1 & $<0.001$ & 0.89 & 0.84 & 0.95 \\
\hline Socioeconomic status & 39.2 & 2 & $<0.001$ & & & & 43.9 & 2 & $<0.001$ & & & \\
\hline Low & 39.0 & 1 & $<0.001$ & 0.32 & 0.22 & 0.46 & 39.8 & 1 & $<0.001$ & 0.33 & 0.23 & 0.46 \\
\hline $\begin{array}{l}\text { Medium } \\
\text { High (ref.) }\end{array}$ & 10.4 & 1 & 0.001 & 0.61 & 0.46 & 0.83 & 3.9 & 1 & 0.049 & 0.74 & 0.55 & 1.00 \\
\hline $\begin{array}{l}\text { Migration background } \\
\text { Yes (ref.) }\end{array}$ & 1.6 & 1 & 0.213 & 1.28 & 0.87 & 1.88 & 11.6 & 1 & 0.001 & 1.90 & 1.31 & 2.74 \\
\hline No & 1.6 & 1 & 0.213 & 1.28 & 0.87 & 1.88 & 11.6 & 1 & 0.001 & 1.90 & 1.31 & 2.74 \\
\hline Residential area & 3.6 & 3 & 0.309 & & & & 11.7 & 3 & 0.008 & & & \\
\hline Rural area & 0.5 & 1 & 0.468 & 1.15 & 0.79 & 1.67 & 8.5 & 1 & 0.003 & 1.78 & 1.21 & 2.61 \\
\hline Small town & 3.5 & 1 & 0.062 & 1.39 & 0.98 & 1.96 & 9.4 & 1 & 0.002 & 1.73 & 1.22 & 2.44 \\
\hline $\begin{array}{l}\text { Medium sized town } \\
\text { City (ref.) }\end{array}$ & 1.1 & 1 & 0.286 & 1.20 & 0.86 & 1.68 & 4.8 & 1 & 0.029 & 1.47 & 1.04 & 2.07 \\
\hline Intercept & 32.4 & 1 & $<0.001$ & 20.83 & & & 4.9 & 1 & 0.027 & 3.02 & & \\
\hline
\end{tabular}

a For boys: $N=938 ; 2 \log$ Likelihood $=1520.1 ;$ Nagelkerke $R^{2}=0.084$

${ }^{\mathrm{b}}$ For girls: $N=890 ; 2 \log$ Likelihood $=1508.8$; Nagelkerke $R^{2}=0.105$

${ }^{\mathrm{c}}$ adj ORadjusted odds ratio

${ }^{\mathrm{d}} C I$ confidence interval; ref. reference value 
chance to be a member of a sports club than girls with a migration background. Adolescent girls living in cities had a $78 \%$ lower chance to be a member of a sports club than girls living in rural areas, a $73 \%$ lower chance than girls living in small towns and a $47 \%$ lower chance than girls living in medium-sized towns, respectively.

Socio-demographic predictors of amount of physical activity in sports clubs

Table 4 shows the results of linear regression analyses predicting the amount of MVPA in sports clubs in 4-10year-old children. The socio-demographic predictors explained $15.4 \%$ of the variance of the amount of MVPA in sports clubs in boys and $7.5 \%$ of that in girls.

Age significantly predicted the amount of MVPA for boys and girls aged 4-10 years. The amount of MVPA increased by $18.0 \mathrm{~min}$ for boys and by $12.3 \mathrm{~min}$ for girls for every additional year of age. Residential area was the only significant predictor in boys. Boys living in rural area and small towns spent 43.0 and 25.6 min less time on MVPA in sports clubs, respectively, than boys living outside of these regions.

Table 5 shows the results of the linear regression analyses for predicting the amount of MVPA in sports clubs in adolescents aged 11 to 17 . The amount of explained variance was very small with $3.6 \%$ for boys and $0.8 \%$ for girls. For both genders, age was the only significant predictor. The amount of MVPA in sports clubs increased by 16.5 and 11.0 min with every year of age for adolescent boys and girls, respectively.

\section{Discussion}

The purposes of this study were to determine representative prevalence rates of sports club membership and the amount of MVPA performed in sports clubs as well as to examine the influence of socio-demographic predictors on sports club membership and the amount of MVPA in sports clubs in children and adolescents in Germany. The results of this study showed that participation in sports in a club setting is an important source of physical activity of children and adolescents in Germany. More than one half of girls and almost two thirds of boys are members of sports clubs. On average, these members exercise $4 \mathrm{~h}$ per week with moderate to high intensity in sports clubs.

In order to achieve the objective that children and adolescents comply with the WHO physical activity guideline of 60 min of MVPA daily (WHO 2010), the setting of sports clubs seems to be an important starting point for children and adolescents in Germany. The results of this study support this assumption. Although members of sports clubs (15.3\%) do not have a significantly higher prevalence of complying with physical activity guidelines than nonmembers (15.1\%), members of sports clubs comply with the physical activity guideline on significantly more days in a week $(M=4.2$ days/week) than nonmembers ( $M=3.6$ days/week). As sports clubs are available and membership is affordable for the majority of the German population, it seems that sports clubs offer considerable potential to raise the level of physical activity of children and adolescents. However, to provide effective nationwide interventions based in sports clubs, understanding socio-demographic predictors of membership and the amount of physical activity is critical.

One predictor of physical activity in sports clubs is gender. It has been shown that boys were significantly more likely to be members of sports clubs and male members exercised significantly more than female members. Gender differences in participation in organised sports and physical activity is one of the most consistent findings in the international physical activity literature (Kimm et al. 2002; Michaud et al. 1999). The gender

Table 4 Linear regression for predicting the time spent in MVPA in sports clubs in 410 year old children

\begin{tabular}{|c|c|c|c|c|c|c|c|c|c|c|}
\hline & \multicolumn{5}{|l|}{ Boys $^{\mathrm{a}}$} & \multicolumn{5}{|l|}{ Girls $^{\mathrm{b}}$} \\
\hline & B & SE & $\beta$ & $t$ & $p$ & $\mathrm{~B}$ & SE & $\beta$ & $t$ & $p$ \\
\hline Age (in years) & 18.0 & 1.6 & 0.37 & 11.3 & $<0.001$ & 12.3 & 1.7 & 0.27 & 7.4 & $<0.001$ \\
\hline $\mathrm{SES}^{\mathrm{c}}$ low & 7.7 & 9.1 & 0.03 & 0.8 & 0.399 & 9.1 & 9.6 & 0.04 & 1.0 & 0.340 \\
\hline SES $^{\mathrm{c}}$ Medium & 2.6 & 6.8 & 0.01 & 0.4 & 0.706 & 2.8 & 7.2 & 0.02 & 0.4 & 0.700 \\
\hline Migration background & 13.4 & 10.5 & 0.04 & 1.3 & 0.202 & 19.1 & 14.4 & 0.05 & 1.3 & 0.187 \\
\hline Rural area & 43.0 & 9.6 & 0.19 & 4.5 & $<0.001$ & 20.1 & 10.9 & 0.08 & 1.8 & 0.067 \\
\hline Small town & 25.6 & 8.5 & 0.13 & 3.0 & 0.003 & 15.3 & 8.9 & 0.08 & 1.7 & 0.085 \\
\hline Medium sized town & 9.4 & 8.3 & 0.05 & 1.1 & 0.261 & 15.5 & 8.8 & 0.08 & 1.8 & 0.078 \\
\hline Intercept & 3.3 & 14.2 & & 0.2 & 0.818 & 15.2 & 14.4 & & 1.1 & 0.290 \\
\hline
\end{tabular}

${ }^{\text {a }}$ For boys: $N=777$; adj. $R^{2}=0.154 ; F_{7.769}=21.1 ; p<0.001$

${ }^{\mathrm{b}}$ For girls: $N=680 ;$ adj. $R^{2}=0.075 ; F_{7.672}=8.8 ; p<0.001$

${ }^{\mathrm{c}} S E S$ socioeconomic status 
Table 5 Linear regression for predicting the time spent in MVPA in sports clubs in 1117 year old adolescents

\begin{tabular}{|c|c|c|c|c|c|c|c|c|c|c|}
\hline & \multicolumn{5}{|l|}{ Boys $^{a}$} & \multicolumn{5}{|l|}{ Girls $^{\mathrm{b}}$} \\
\hline & $\mathrm{B}$ & $\mathrm{SE}$ & $\beta$ & $t$ & $p$ & B & SE & $\beta$ & $t$ & $p$ \\
\hline Age (in years) & 16.5 & 3.5 & 0.20 & 4.7 & $<0.001$ & 11.0 & 4.0 & 0.14 & 2.8 & 0.006 \\
\hline $\mathrm{SES}^{\mathrm{c}}$ low & 1.6 & 20.8 & 0.00 & 0.1 & 0.939 & 13.3 & 23.8 & 0.03 & 0.6 & 0.577 \\
\hline $\mathrm{SES}^{\mathrm{c}}$ medium & 11.2 & 15.4 & 0.03 & 0.7 & 0.467 & 8.3 & 18.1 & 0.03 & 0.5 & 0.648 \\
\hline Migration background & 26.7 & 24.5 & 0.05 & 1.1 & 0.277 & 7.3 & 27.9 & 0.01 & 0.3 & 0.793 \\
\hline Rural area & 4.2 & 21.9 & 0.01 & 0.2 & 0.847 & 3.8 & 25.9 & 0.01 & 0.1 & 0.885 \\
\hline Small town & 30.7 & 19.6 & 0.09 & 1.6 & 0.118 & 14.1 & 23.6 & 0.04 & 0.6 & 0.552 \\
\hline Medium sized town & 6.4 & 19.3 & 0.02 & 0.3 & 0.740 & 3.3 & 24.1 & 0.01 & 0.1 & 0.892 \\
\hline Intercept & 11.383 & 53.787 & & 0.2 & 0.832 & 31.9 & 61.2 & & 0.5 & 0.603 \\
\hline
\end{tabular}

${ }^{\text {a }}$ For boys: $N=550$; adj. $R^{2}=0.036 ; F_{7.542}=3.9 ; p<0.001$

${ }^{\mathrm{b}}$ For girls: $N=414$; adj. $R^{2}=0.008 ; F_{7.406}=1.5 ; p=0.173$

${ }^{\mathrm{c}} S E S$ socioeconomic status

differences in organised sports might reflect cultural values, structural circumstances and reward systems that encourage participation by males (Vilhjalmsson and Kristjansdottir 2003).

The importance of gender in the context of physical activity in sports clubs is further shown by the fact that the influence of other socio-demographic predictors depended on gender as, for instance, demonstrated by the influence of age on physical activity in sports clubs. In girls, the prevalence of being a member in a sports club during childhood and early adolescence remained constant at $50 \%$. However, in late adolescence, the proportion of female members of sports clubs decreased. Comparable declines in physical activity in girls in late adolescence have been reported by Kimm et al. (2002). In boys, there is a steep and continuous increase in membership during childhood and a continuous decrease during adolescence. These results indicate that the importance of performing sports in clubs increases during childhood and early adolescence for boys, whereas it remains constant for girls in the same age period, with a higher drop-out rate in late adolescence. Similar decreases in sport participation during adolescents were also found in other European countries (Gracia-Marco et al. 2010; Telama and Yang 2000; Michaud et al. 1999) and the United States (Pate et al. 2000). In late adolescence, the role of sports in clubs in everyday life decreases presumably because of increasing school demands (e.g. long school days, afternoon school, homework) in boys and girls. Rowland (Rowland 1999) suggested a decrease of the biological drive for physical activity and increasing independence during adolescence that allows teenagers to pursue their own lifestyles.

However, those who remained members of sports clubs continuously increased the amount of MVPA with every year of age during childhood and adolescences for both boys and girls. Comparable observations have been reported for other European countries (Seabra et al. 2007). It appears that the key to maintaining higher levels of physical activity lies in maintaining membership in sports clubs, and that a meaningful intervention could be targeted at increasing the membership numbers in sports clubs. However, these results could also mean that members of sports clubs become more active because only those who compete on a higher level that requires more training, and therefore more physical activity, remain members. Therefore, sports clubs should offer programs for less competitive adolescents to counteract the decrease of membership numbers in adolescence. However, longitudinal studies are needed to clarify this issue.

Socioeconomic status seems to be a persistent predictor of membership in sports clubs. Children and adolescents with higher socioeconomic status have a higher probability of being a member in sports clubs than participants with lower socioeconomic status. Hence, social disparity determines participation in sports related physical activity as was shown in several international studies (Gracia-Marco et al. 2010; La Torre et al. 2006; Pitsavos et al. 2005; Fernandes et al. 2012). However, among members in sports clubs, their socioeconomic status does not influence their amount of physical activity. Therefore, interventions should focus to facilitate access to sports clubs for families with low income.

Migration background seems to be a stronger predictor of membership in sports clubs in girls than in boys. Especially during childhood, the effect of a migration background is very strong for girls. Presumably, integration problems of girls with a migration background are especially evident in childhood and tend to decrease during adolescence. However, among members of sports clubs, migration background does not influence the amount of MVPA. A possible explanation could be that families with a migration background (e. g. with an Islamic background) traditionally do not support girls' participation in sports. However, the present study does not provide the required data to test this hypothesis. 
Residential area has a differential effect on physical activity in sports clubs. In girls, residential area is a significant predictor of sports club membership and in boys a significant predictor of the amount physical activity in sports clubs. During childhood, living in a rural area negatively affects the probability of being a member in sports clubs in girls and the amount of physical activity in boys. It is possible that the less developed infrastructure of sports clubs (e.g. long distances to sports clubs) in rural areas is responsible for the negative effects in children. However, during adolescence, girls living in cities are less likely to be members of sports clubs. The availability of a broad range of alternative leisure time activities in cities (e.g. music clubs, cinemas, theatres etc.) may distract from being physically active in sports clubs in adolescent girls. These results imply that the role of residential area for being a member in sports clubs changes with age.

The major strength of this study is that the results are based on national level representative data for children and adolescents. However, the results of this study should be interpreted with caution because of some limitations. First, this study has a cross-sectional design, and hence the results of the study do not allow causal inferences of predictors. For example, age and cohort effects cannot be distinguished in cross-sectional studies. Second, the results were based on self-reported data which may lead to an overestimation of physical activity in sports clubs. Third, this study did not provide information on school obligations, attitudes of parents, and infrastructure of sports clubs that would allow for a better understanding of the effects of socio-demographic variables.

\section{Conclusion}

The results of this study indicate that physical activity in sports clubs is wide-spread among children and adolescents in Germany. While membership in sports clubs depends on sociodemographic correlates, the amount of physical activity only depends on age and gender and partially on residential area. Interventions to increase general physical activity level should focus on increasing the number of members in sports clubs. To some extent, access to sports clubs seems to be determined by social status. Hence, state and communal policies should try to make access to sports clubs easier (e.g. by supporting families with low income with coupons for membership in sports clubs). During childhood, boys and girls with migration backgrounds should be targeted by interventions to integrate them into the sports clubs system. Adolescence seems to be a critical stage of life for discontinuing sports club participation. Special programs in sports clubs (e.g. cooperation between school and sports clubs) should be developed to meet the specific needs of these age groups.
Acknowledgements This study was funded by a project grant from the German Bundesministerium für Bildung und Forschung (Federal Minis try of Education and Research) and by the German Bundesministerium für Gesundheit (Federal Ministry of Health). We thank all the adolescents who participated in this study. We would also like to express our appre ciation to PD Dr. Annegret Mündermann (University of Konstanz) and Dr. Stefanie Everke Buchanan (Zeppelin University Friedrichshafen/Uni versity of Konstanz) for their writing assistance on behalf of the authors. The second author thanks the Ministry of Science of the German State of Baden Württemberg for supporting this project through a grant scholar ship of the Brigitte Schlieben Lange Programm.

Conflicts of interest The authors declare that they have no conflict of interest.

\section{References}

Baur J, Burrmann U (2000) Unerforschtes Land: Jugendsport in ländlichen Regionen [Unexplored country: youth sports in rural regions]. Meyer \& Meyer, Aachen, Germany

Bös K (2009) Motorik Modul: Eine Studie zur motorischen Leistungs fähigkeit und körperlich sportlichen Aktivität von Kindern und Jugendlichen in Deutschland. Abschlussbericht zum Forschungs projekt [Motorik modul: a study on motor abilities und physical activity of children and adolescents in Germany. Final report of the research project.]. Nomos, Baden Baden, Germany

Bouchard C, Blair SN, Haskell WL (2012) Physical activity and health. Human Kinetics, Champaign, IL

Brettschneider W (2001) Effects of sport club activities on adolescent development in Germany. Eur J Sport Sci 1(2):1 11. doi:10.1080/ 17461390100071201

Breuer C, Wicker P (2009) Die Situation der Sportvereine in Deutsch land: ein Überblick. In: Breuer C (ed) Sportentwicklungsbericht 2007/2008: Analyse zur Situation der Sportvereine in Deutsch land. Sportverlag Strauß, Köln, Germany, pp 2648

Dobbinson SJ, Hayman JA, Livingston PM (2006) Prevalence of health promotion policies in sports clubs in Victoria, Australia. Health Promot Int 21(2):121 129. doi:10.1093/heapro/dak001

Fernandes RA, Reichert FF, Monteiro HL, Freitas Júnior IF, Cardoso JR, Ronque ERV, de Oliveira AR (2012) Characteristics of family nucleus as correlates of regular participation in sports among ado lescents. Int J Public Health 57(2):431 435. doi:10.1007/s00038 01002077

Gracia Marco L, Tomas C, Vicente Rodriguez G, Jimenez Pavon D, Rey Lopez JP, Ortega FB, Lanza Saiz R, Moreno LA (2010) Extra curricular participation in sports and socio demographic factors in Spanish adolescents: the AVENA Study. J Sports Sci 28(13):1383 1389. doi:10.1080/02640414.2010.510846

Heinemann K (1999) Sports clubs in Europe. In: Heinemann K (ed) Sport clubs in various European countries. Schattauer, Schorndorf, Germany, pp 1332

Jekauc D, Reimers AK, Wagner MO, Woll A (2012) Prevalence and socio demographic correlates of the compliance with the physical activity guidelines in children and adolescents in Germany. BMC Publ Health 12(1):714. doi:10.1186/1471 245812714

Jekauc D, Wagner MO, Kahlert D, Woll A (2013) Reliabilität und Validität des MoMo Aktivitätsfragebogens für Jugendliche (MoMo AFB) [Reliability and validity of MoMo physical activity questionnaire for adolescents (MoMo AFB)]. Diagnostica 59(2):1 12. doi:10.1025/0012 1924/a000083

Kamtsiuris P, Lange M, Schaffrath Rosario A (2007) Der Kinder und Jugendgesundheitssurvey (KiGGS): Stichprobendesign, Response 
und Nonresponse Analyse [The German Health Interview and Exam ination Survey for Children and Adolescents (KiGGS): sample design, response and nonresponse analysis]. Bundesgesundheitsbla Gesundheitsforsch Gesundheitsschutz 50(5 6):547 556. doi:10.1007/ s001030070215 9

Kimm SYS, Glynn NW, Kriska AM, Barton BA, Kronsberg SS, Dan iels SR, Crawford PB, Sabry ZI, Liu K (2002) Decline in physical activity in black girls and white girls during adolescence. N Engl J Med 347(10):709 715. doi:10.1056/NEJMoa003277

Kokko S, Kannas L, Villberg J (2009) Health promotion profile of youth sports clubs in Finland: club officials' and coaches' percep tions. Health Promot Int 24(1):26 35. doi:10.1093/heapro/dan040

Kurth BM (2007) Der Kinder und Jugendgesundheitssurvey (KiGGS): ein Überblick über Planung, Durchführung und Ergebnisse unter Berücksichtigung von Aspekten eines Qualitätsmanagements [The German Health Interview and Examination Survey for Children and Adolescents (KiGGS): an overview of its planning, implementation and results taking into account aspects of quality management]. Bundesgesundheitsbla Gesundheitsforsch Gesundheitsschutz 50(5 6):533 546. doi:10.1007/s00103007 $0214 \mathrm{x}$

Kurth BM, Kamtsiuris P, Holling H, Schlaud M, Dolle R, Ellert U, Kahl H, Knopf H, Lange M, Mensink GB, Neuhauser H, Rosario AS, Scheidt Nave C, Schenk L, Schlack R, Stolzenberg H, Thamm M, Thierfelder W, Wolf U (2008) The challenge of comprehensively mapping children's health in a nation wide health survey: design of the German KiGGS Study. BMC Publ Health 8:196. doi:10.1186/1471 24588196

La Torre G, Masala D, De Vito E, Langiano E, Capelli G, Ricciardi W (2006) Extra curricular physical activity and socioeconomic status in Italian adolescents. BMC Publ Health 6(1):22. doi:10.1186/ 14712458622

Michaud P, Narring F, Cauderay M, Cavadini C (1999) Sports activity, physical activity and fitness of 9 to 19 year old teenagers in the canton of Vaud (Switzerland). Schweiz Med Wochenschr 129(18):691 699

Pate RR, Trost SG, Levin S, Dowda M (2000) Sports participation and health related behaviors among US youth. Arch Pediatr Adolesc Med 154(9):904 911. doi:10.1001/archpedi.154.9.904
Pitsavos C, Panagiotakos D, Lentzas Y, Stefanadis C (2005) Epidemi ology of leisure time physical activity in socio demographic, life style and psychological characteristics of men and women in Greece: the ATTICA study. BMC Publ Health 5(1):37. doi:10.1186/ 14712458537

Rowland TW (1999) Adolescence: a "risk factor" for physical inactivity. President's Council Physical Fitness Sports Res Digest 3(6):1 8

Seabra AF, Mendonça DM, Thomis MA, Malina RM, Maia JA (2007) Sports participation among Portuguese youth 10 to 18 years. J Phys Act Health 4(4):370 380

Strong WB, Malina RM, Blimkie CJR, Daniels SR, Dishman RK, Gutin B, Hergenroeder AC, Must A, Nixon PA, Pivarnik JM (2005) Evidence based physical activity for school age youth. J Pediatr 146(6): 732 737. doi:10.1016/j.jpeds.2005.01.055

Telama R, Yang X (2000) Decline of physical activity from youth to young adulthood in Finland. Med Sci Sports Exerc 32(9):1617 1622. doi:10.1097/00005768 20000900000015

Telama R, Yang X, Viikari J, Välimäki I, Wanne O, Raitakari O (2005) Physical activity from childhood to adulthood: a 21 year tracking study. Am J Prev Med 28(3):267 273. doi:10.1016/ j.amepre.2004.12.003

Van der Horst K, Paw M, Twisk JWR, Van Mechelen W (2007) A brief review on correlates of physical activity and sedentariness in youth. Med Sci Sports Exerc 39(8):1241 1250. doi:10.1249/ mss.0b013e318059bf35

Vilhjalmsson R, Kristjansdottir G (2003) Gender differences in physical activity in older children and adolescents: the central role of organized sport. Soc Sci Med 56(2):363 374. doi:10.1016/ S0277 9536(02)00042 4

WHO (2010) Global recommendations on physical activity for health. World Health Organisation, Geneva

Winkler J, Stolzenberg H (1999) Der sozialschichtindex im bundes gesundheitssurvey [Social class index in the federal health survey]. Gesundheitswesen 61:S178 183

Woll A, Kurth BM, Opper E, Worth A, Bos K (2011) The "Motorik Modul" (MoMo): physical fitness and physical activity in German children and adolescents. Eur J Pediatr 170(9):1129 1142. doi:10.1007/s00431 01013914 